\title{
Systematic risk analysis: first steps towards a new definition of beta
}

\author{
Michel FLIESS ${ }^{*, * *} \quad$ Cédric JOIN ${ }^{*, * * *}$ \\ ${ }^{*}$ INRIA-ALIEN \\ ** LIX (CNRS, UMR 7161), École polytechnique \\ 91128 Palaiseau, France \\ Michel.Fliess@polytechnique.edu \\ *** CRAN (CNRS, UMR 7039), Nancy-Université \\ BP 239, 54506 Vandœuvre-lès-Nancy, France \\ Cedric. Join@cran.uhp-nancy.fr
}

\begin{abstract}
We suggest a new model-free definition of the beta coefficient, which plays an important rôle in systematic risk management. This setting, which is based on the existence of trends for financial time series via nonstandard analysis (Fliess M., Join C.: A mathematical proof of the existence of trends in financial time series, Proc. Int. Conf. Systems Theory: Modelling, Analysis and Control, Fes, 2009, online: http://hal.inria.fr/inria-00352834/en/) leads to convincing computer experiments which are easily implementable.
\end{abstract}

Keywords: Quantitative finance, risk analysis, beta, alpha, trends, technical analysis, estimation techniques, forecasting, abrupt changes, nonstandard analysis.

\section{INTRODUCTION}

This communication studies systematic (or market) risks in quantitative finance via the well known $\beta$ coefficient (see, e.g., $[3 ; 25]$ ), which plays a key rôle since the introduction of the capital asset pricing model (CAPM) for portfolio management by Sharpe [24] and others. The following definition in plain words of $\beta$ is borrowed from Lhabitant [18]: "Another interesting risk measure is called beta and is denoted by the Greek symbol $\beta$. Simply stated, $\beta$ measures how risky a fund may be as compared to the overall stock market, typically approximated to the Standard and Poor's 500 or the MSCI World. A fund that moves in harmony with the market is said to have a beta of 1.0. Other things being equal, if the market goes up $10 \%$, the fund is expected to go up up $10 \%$. If the market goes down $10 \%$, the fund is expected to go down up $10 \%$. When a fund has a beta that is less than 1, it is supposed to move less in price than the market in general. Conversely, a fund with a beta higher than 1 is supposed to move more in price than the market in general. Hence, beta measures the risk of a fund by telling us how much its market price changes compared to changes in the overall stock market. A fund with a beta of more than 1 tends be riskier than the market, while a fund with a beta of less than 1 is less risky." The utilization of $\beta$ for the purpose of $\beta$ neutrality and hedging has become important for investments, including the hedge fund industry (see, e.g., $[17 ; 18 ; 19 ; 20]$ and the references therein).

The linear character of the $\beta$ coefficient explains some of its shortcomings (see, e.g., [7;8] and the references therein).
The advantage of taking a time-varying $\beta$ does not seem to have been yet clearly demonstrated (see, e.g., $[1 ; 4]$ ). A similar conclusion holds for the replacement of linear statistical regressions by nonlinear ones (see, e.g., [18; 20] and the references therein). We follow here another route, which is based on recent advances in automatic control and in signal processing:

(1) The Cartier-Perrin theorem [6] may be understood as a nonstandard definition of noises [9]. It yields noise attenuation [9] without having recourse to any probabilistic and statistical tool. It implies, therefore, the existence of trends in financial time series [11]. A crucial assumption in technical analysis, which is quite popular among many traders (see, e.g., [2; 23] and $[3 ; 19])$, is thus confirmed.

(2) A powerful derivatives estimation of noisy signals has been achieved by real-time techniques which are of algebraic flavor $[15 ; 22]$. Applications to new indicators in technical analysis have already been explored in $[11 ; 13]$.

(3) New techniques for model-free control [10; 12] have been successfully applied in several concrete situations (automotive industry, smart material actuators, hydroelectric power plants, ... ). ${ }^{1}$ It shows that the necessity of writing down a "precise" mathematical model for controlling "complex" systems has been largely exaggerated in today's academic literature.

Our paper is organized as follows. Section 2 after a brief review of the existence of trends via the Cartier-Perrin theorem introduces a model-free definition of $\beta$ and $\alpha$. Section 3 sketches the techniques which are necessary for estimating beta and some related quantities. The numerous computer experiments in Section 4 show reliable forecasts of beta and of its abrupt changes via our techniques, which are easily implementable.

Analogous model-free methods will be developed in a near future in order to present various hedging strategies (see, e.g., $[3 ; 25])$, and a large range of highly efficient tools for risk analysis, which again will be easily implementable.

\footnotetext{
1 See some references in $[10 ; 12]$. All references to applications of model-free control, to which the authors are associated, are or will be soon available at http://hal.inria.fr.
} 


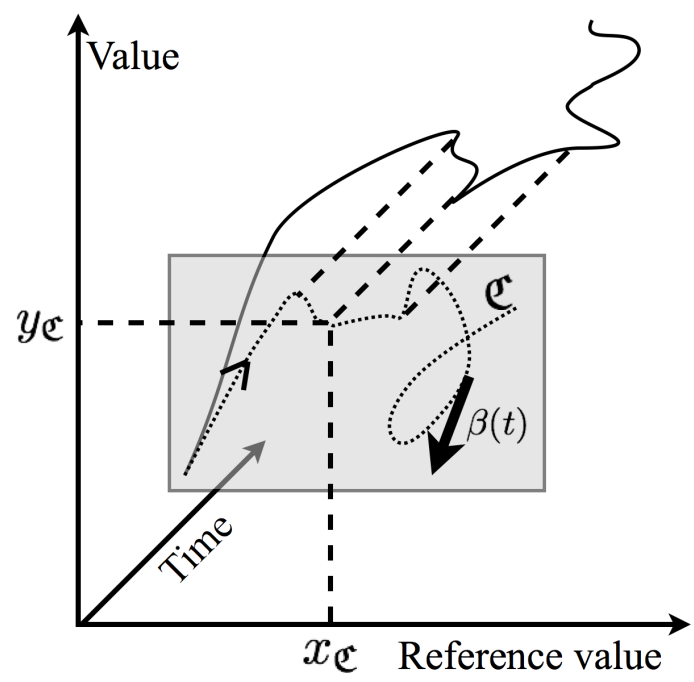

Figure 1. 2D-3D illustration

\section{A MODEL-FREE DEFINITION OF BETA}

\subsection{Quick fluctuations and trends in financial time series}

Consider a time series $f(t)$. The Cartier-Perrin theorem [6], which is expressed in the language of nonstandard analysis, ${ }^{2}$ states that, under a very mild integrability condition, there exists the following additive decomposition of $f(t)$ :

$$
f(t)=f_{\text {trend }}(t)+f_{\text {fluctuation }}(t)
$$

where $f_{\text {trend }}(t)$ and $f_{\text {fluctuation }}(t)$ are respectively the trend and a quickly fluctuating function around 0 :

- the quickly fluctuating function $f_{\text {fluctuation }}(t)$ is defined by the fact that its integral $\int_{a}^{b} f_{\text {fluctuation }}(\tau) d \tau$ over any finite interval $[a, b]$ is infinitesimal, i.e., "very small";

- the trend $f_{\text {trend }}(t)$ is integrable and almost everywhere continuous.

Those quick fluctuations are analogous to corrupting additive noises in engineering, and especially in signal processing and in automatic control. The practical calculation of the trend in $[11 ; 13]$ is therefore achieved via estimation techniques which were developed elsewhere and for other purposes $[15 ; 22]$. Those techniques may be viewed as an extension of the familiar moving average methods which are central in technical analysis (see, e.g., $[2 ; 23]$ ).

\subsection{Beta}

Take two time series $X(t)$ and $Y(t)$, which satisfy the integrability assumptions of the Cartier-Perrin theorem. Assume moreover that the corresponding trends $X_{\text {trend }}(t)$ and $Y_{\text {trend }}(t)$ are locally smooth enough, i.e., they are locally $\mathcal{C}^{k}$ for $k$ large enough. Consider the space curve $t, X_{\text {trend }}(t), Y_{\text {trend }}(t)$ in the Euclidean space with coordinates $t, x, y$. Its projection on the $x, y$ plane is the plane curve $\mathfrak{C}$ defined by

$$
x_{\mathfrak{C}}(t)=X_{\text {trend }}(t), y_{\mathfrak{C}}(t)=Y_{\text {trend }}(t)
$$

${ }^{2}$ See, e.g., [11] and especially [21] for a general presentation and related references.
The tangent of $\mathfrak{C}$ at a regular point, which is defined by $\frac{d X_{\text {trend }}(t)}{d t}, \frac{d Y_{\text {trend }}(t)}{d t}$, yields, if $\frac{d X_{\text {trend }}(t)}{d t} \neq 0$,

$$
\Delta y_{\mathfrak{C}} \approx \beta(t) \Delta x_{\mathfrak{C}}
$$

where

- $\Delta x_{\mathfrak{C}}=x_{\mathfrak{C}}(t+h)-x_{\mathfrak{C}}(t), \Delta y_{\mathfrak{C}}=y_{\mathfrak{C}}(t+h)-y_{\mathfrak{C}}(t)$

- $h \in \mathbb{R}$ is "small";

$$
\beta(t)=\frac{\frac{d Y_{\text {trend }}}{d t}}{\frac{d X_{\text {trend }}}{d t}}
$$

When $y_{\mathfrak{C}}(t)$ may be viewed locally as a smooth function of $x_{\mathfrak{C}}(t)$, Equation (3) becomes

$$
\beta(t)=\frac{d y_{\mathfrak{C}}}{d x_{\mathfrak{C}}}
$$

With the original time series $X(t), Y(t)$, Equation (1) shows that Equation $(2)$ becomes

$$
\Delta Y \approx \beta(t) \Delta X+F_{\Delta}(t)
$$

where

- $\Delta X=X(t+h)-X(t), \Delta Y=Y(t+h)-X(t)$;

- $F_{\Delta}(t)$ is a quickly fluctuating function [6].

Remark 2.1. Equations (2) and (5) relate obviously the amplitudes of the variations of $X$ and $Y$ during a "short" time interval. Let us emphasize that our definition of $\beta$ is completely independent of any a priori mathematical modeling ${ }^{3}$ since some mild smoothness assumptions are the only claims on the data.

Remark 2.2. Our $\beta$ is, to the best of our knowledge, the first quantitative attempt to approach intermarket technical analysis (see, e.g., [2; 23]).

Remark 2.3. The behavior of $\beta(t)$ will also be related to higher order derivatives (see Section 4).

\subsection{Alpha}

When Equation (4) holds, it yields the following straightforward definition of the $\alpha$ coefficient:

$$
y_{\mathfrak{C}}=\alpha_{x_{I}}+\int_{x_{I}}^{x_{\mathfrak{C}}} \beta d x
$$

We recover a CAPM-like formula for $\alpha$ and $\beta$ (see, e.g., $[3 ; 25])$, i.e., $y_{\mathfrak{C}}=\alpha_{0}+\beta x_{\mathfrak{C}}$, if

- $\beta$ is constant, i.e., $\mathfrak{C}$ is a straight line;

- $x_{I}=0$.

Remark 2.4. A more intrinsic definition may be deduced from the geometry of the curve $\mathfrak{C}: \alpha_{x_{I}}$ is the ordinate of point of abscissa $x=x_{I}$. Note however that this point may not be unique.

\section{ESTIMATION TECHNIQUES}

\subsection{An integral formula for derivative estimation}

Consider again the time series $f(t)$ of Section 2.1. When $f_{\text {trend }}(t)$ in Equation (1) is smooth enough, elementary

\footnotetext{
3 Probabilistic and statistical modeling belong of course to mathematical modeling (see, e.g., the enlightening discussion in [5]). Let us emphasize once again that we do not need any probabilistic and/or statistical framework for taking into account the uncertainty in finance (see the comments in $[11 ; 13]$ ).
} 
algebraic manipulations (see, e.g., [22] and [16]) show that, for $i \geq 0$,

$$
\frac{d^{i} f_{\text {trend }}}{d t^{i}}(t) \approx \int_{t-T}^{t} P_{i}(\tau) f(\tau) d \tau
$$

where

- $T>0$ is the evaluation time window, which is usually quite "short";

- $P$ is a polynomial function;

- when $i=0$ (resp. $i \supsetneqq 0$ ) we obtain an estimation of the trend (resp. of the derivative of order $i$ of the trend).

Remark 3.1. This setting, which is extremely useful in automatic control and signal processing (see, e.g., $[15 ; 16]$ and the references therein), has already been employed in financial engineering for questions related to technical analysis $[11 ; 13]$.

\subsection{Estimating beta}

The division in Equation (3) leads to numerical instabilities when applying Formula (7). The numerical implementation of the integral Formula (7) for estimating $\beta$ via Equation (4) does therefore necessitate some care when compared to what has already been achieved in the past (see [22] and [16]):

- the function $x_{\mathfrak{C}}(t)$ does not necessarily increase or decrease in a monotonic way,

- the sampling with respect to $x_{\mathfrak{C}}$ is not necessarily uniform.

Practical details will be provided elsewhere.

Remark 3.2. Similar calculations may be derived for estimating higher order derivatives like in Remark 2.3.

\section{COMPUTER EXPERIMENTS}

We compare with the S\&P 500 index three quite different assets:

(1) JPMORGAN CHASE (JPM) from 1983-12-30 until 2009-07-21 (6267 days),

(2) IBM from 1962-01-02 to 2009-07-21 (11776 days),

(3) COCA COLA (CCE) from 1986-11-24 until 2009-0721 (5519 days).

Figure 2 displays the prices, which are normalized for an obvious comparison's sake, and their corresponding trends which are obtained as in $[11 ; 13]$.

Consider, as in Section 2.2, the projections in the $x, y$ plane. We exhibit the values of $\beta$ and $\frac{d \beta}{d x}$, and their 20 days forecasts, which are obtained via similar extrapolation techniques to those in $[11 ; 13]$. The various Figures show that the predictions are quite good.

An abrupt change of $\beta$ is a "sudden" and "important" variation of its values. Thanks to techniques in [13; 14], Figure 3 -(d) displays the 20 days forecasts of abrupt changes of $\beta$ for JPM. The results are again quite impressive.

Remark 4.1. It is noteworthy that $\beta$ is "almost" constant during large time intervals. According to Section 2.3, our simulations do confirm to some extent the well known CAPM modeling (see, e.g., [3; 25]). This traditional setting nevertheless is unable to deal with abrupt changes.
Remark 4.2. The adaptation of our techniques to high frequency finance would be quite easy.

\section{REFERENCES}

[1] Adrian T., Franzoni F.: Learning about beta: Time-varying factor loadings, expected returns, and the conditional CAPM, J. Empirical Finance, 16, 537-556, 2009.

[2] Béchu T., Bertrand E., Nebenzahl J.: L'analyse technique (6 ${ }^{e}$ éd.), Economica, 2008.

[3] Bodie Z., Kane A., Marcus A.J.: Investments ( $7^{\text {th }}$ ed.), McGraw-Hill, 2008.

[4] Bollerslev T., Engle R., Wooldridge J.: A capital asset pricing model with time varying covariances, J. Political Economy, 96, 116-131, 1988.

[5] Bouleau N.: Mathématiques et risques financiers, Odile Jacob, 2009.

[6] Cartier P., Perrin Y.: Integration over finite sets, in Nonstandard Analysis in Practice, F. \& M. Diener (Eds), Springer, 1995, pp. 195-204.

[7] Fernández P.: Are calculated betas good for anything?, Working Paper, 2008 (available at http://ssrn.com/abstract=504565).

[8] Fernández P.: 201 Errores en la Valoración de Empresas, Ediciones Deusto, 2008.

[9] Fliess M.: Analyse non standard du bruit, C.R. Acad. Sci. Paris Ser. I, 342, 797-802, 2006.

[10] Fliess M., Join C.: Commande sans modèle et commande à modèle restreint, e-STA, 5 ( $\left.\mathrm{n}^{\circ} 4\right), 1-23,2008$, (available at http://hal.inria.fr/inria-00288107/en/).

[11] Fliess M., Join C.: A mathematical proof of the existence of trends in financial time series, in Systems Theory: Modeling, Analysis and Control, A. El Jai, L. Afifi, E. Zerrik (Eds), Presses Universitaires de Perpignan, 2009, pp. 43-62 (available at http://hal.inria.fr/inria-00352834/en/).

[12] Fliess M., Join C.: Model-free control and intelligent PID controllers: towards a possible trivialization of nonlinear control?, $15^{\text {th }}$ IFAC Symp. System Identif., Saint-Malo, 2009 (available at http://hal.inria.fr/inria-00372325/en/).

[13] Fliess M., Join C.: Towards new technical indicators for trading systems and risk management, $15^{\text {th }}$ IFAC Symp. System Identif., Saint-Malo, 2009 (available at http://hal.inria.fr/inria-00370168/en/).

[14] Fliess M., Join C., Mboup M.: Algebraic change-point detection, Applicable Algebra Engin. Communic. Comput., 2010 (soon available at http://hal.inria.fr/).

[15] Fliess M., Join C., Sira-Ramírez H.: Non-linear estimation is easy, Int. J. Model. Identif. Control, 4, 12-27, 2008 (available at http://hal.inria.fr/inria-00158855/en/).

[16] García Collado F.A., d'Andréa-Novel B., Fliess M., Mounier H.: Analyse fréquentielle des dérivateurs algébriques, XXII ${ }^{e}$ Coll. GRETSI, Dijon, 2009 (available at http://hal.inria.fr/inria-00394972/en/).

[17] Jaeger L.: Alternative Beta Strategies and Hedge Fund Replication, Wiley, 2008.

[18] Lhabitant F.-S.: Hedge Funds: Quantitative Insights, Wiley, 2004.

[19] Lhabitant F.-S.: Handbook of Hedge Funds, Wiley, 2006.

[20] Lo A.W.: Hedgefunds: An Analytic Perspective, Princeton University Press, 2008.

[21] Lobry C., Sari T.: Nonstandard analysis and representation of reality, Int. J. Control, 81, 517-534, 2008.

[22] Mboup M., Join C., Fliess M.: Numerical differentiation with annihilators in noisy environment, Numer. Algor., 50, 439467, 2009.

[23] Murphy J.J.:Technical Analysis of the Financial Markets: A Comprehensive Guide to Trading Methods and Applications ( $2^{\text {nd }}$ ed.), New York Institute of Finance, 1999.

[24] Sharpe W.F.: Capital asset prices: a theory of market equilibrium under conditions of risk, J. Finance, 19, 425-442, 1964.

[25] Vernimmen P., Quiry P., Le Fur Y.: Finance d'entreprise ( $8^{e}$ éd.), Dalloz, 2009. 


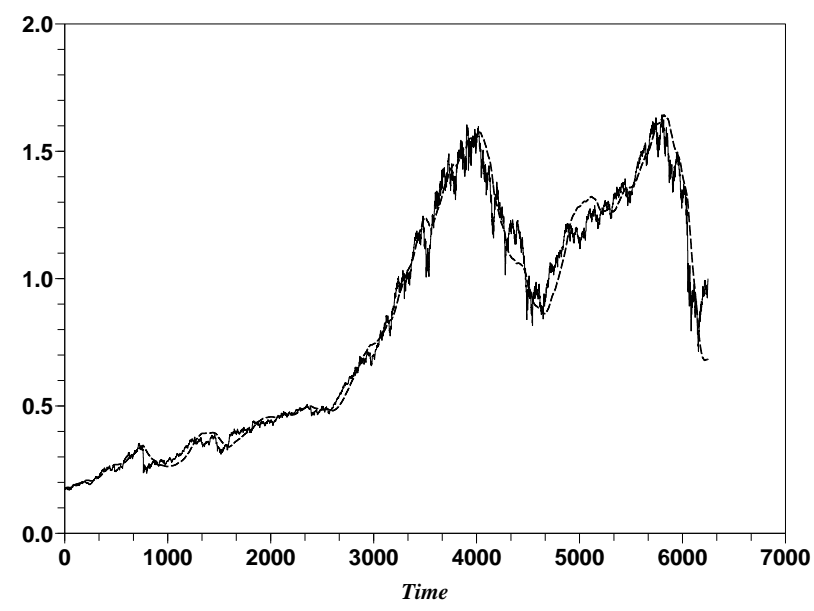

(a) S\&P 500 normalized (-) and its trend (- -)

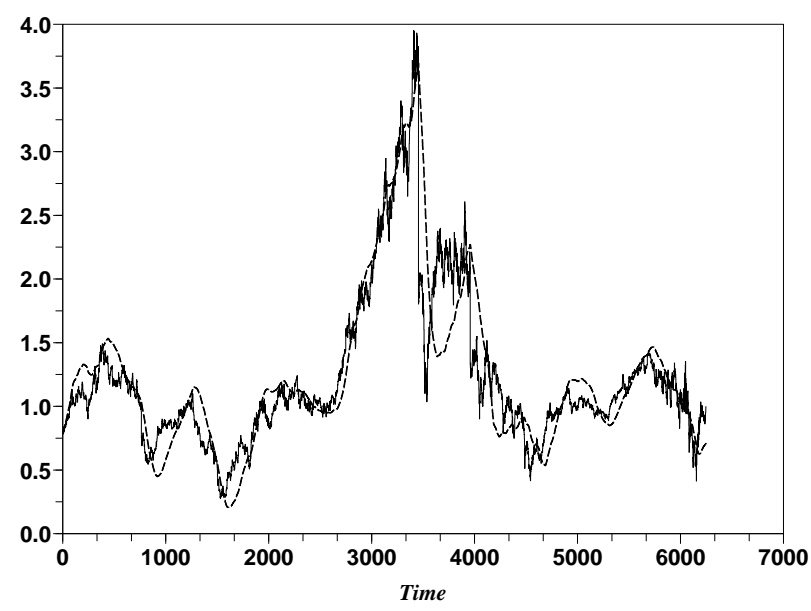

(c) JPM normalized (-) and its trend (- -)

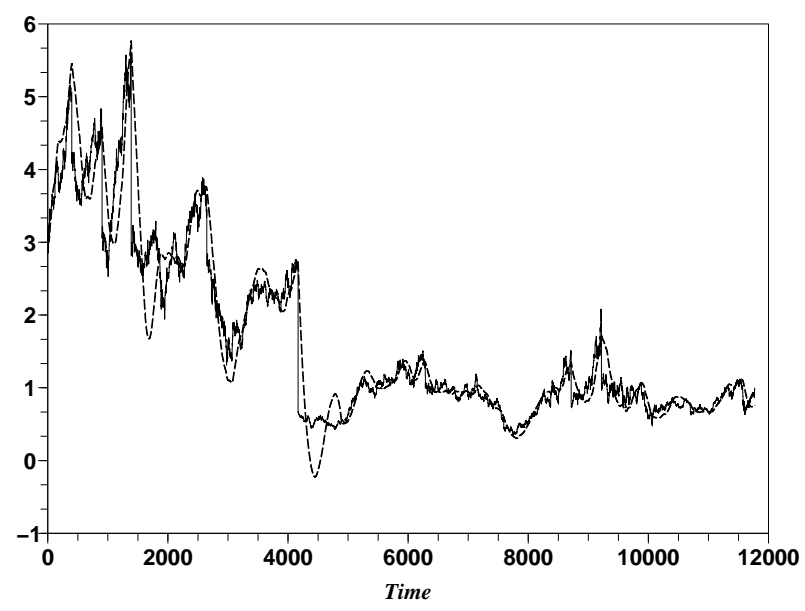

(b) IBM normalized ,-) and its trend (- -)

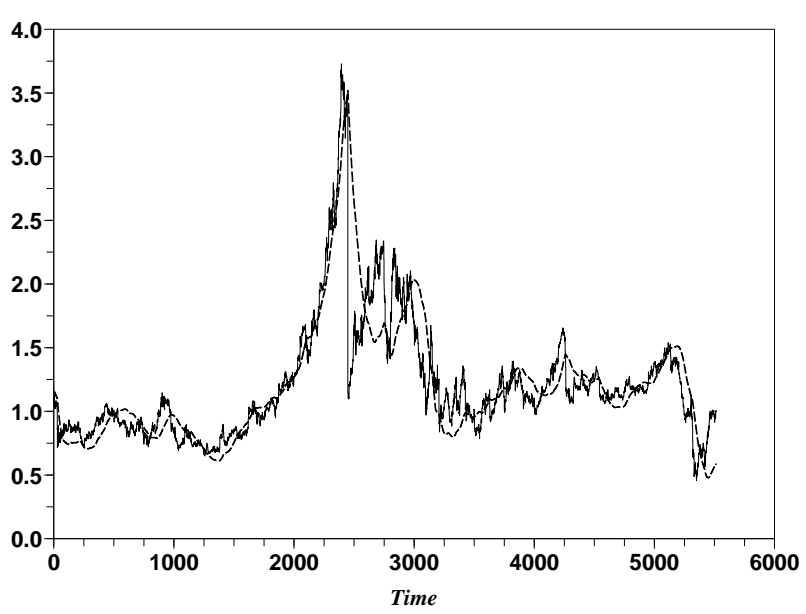

(d) CCE normalized (-) and its trend (- -)

Figure 2. Different assets 


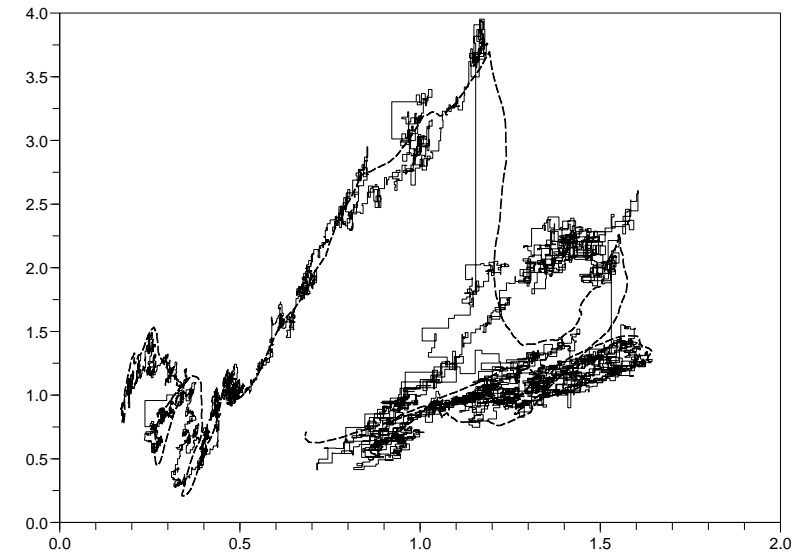

(a) $\mathfrak{C}$ : JPM (S\&P 500) (-) and its trend (- -)

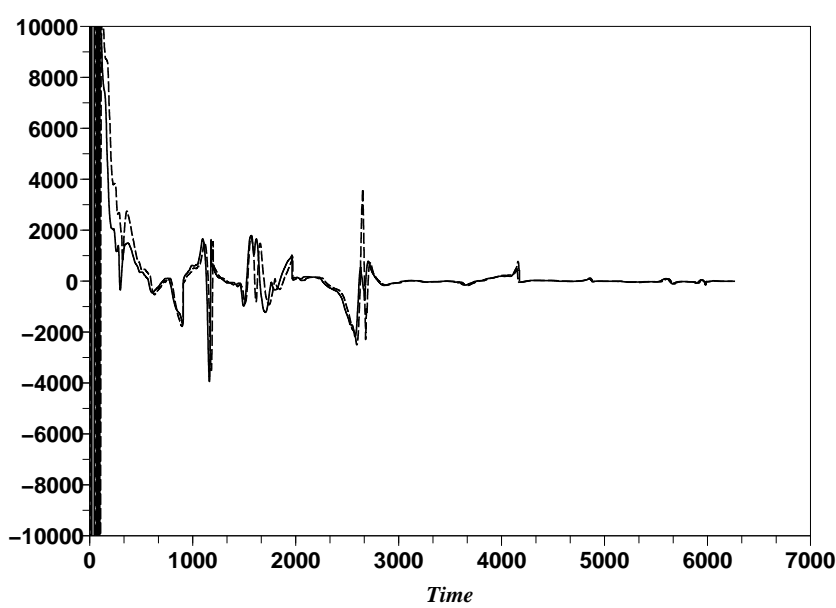

(c) JPM's $\frac{d \beta}{d x_{\mathfrak{C}}}(-)$ and 20 days forcasting (- -)

Figure 3. JPM case

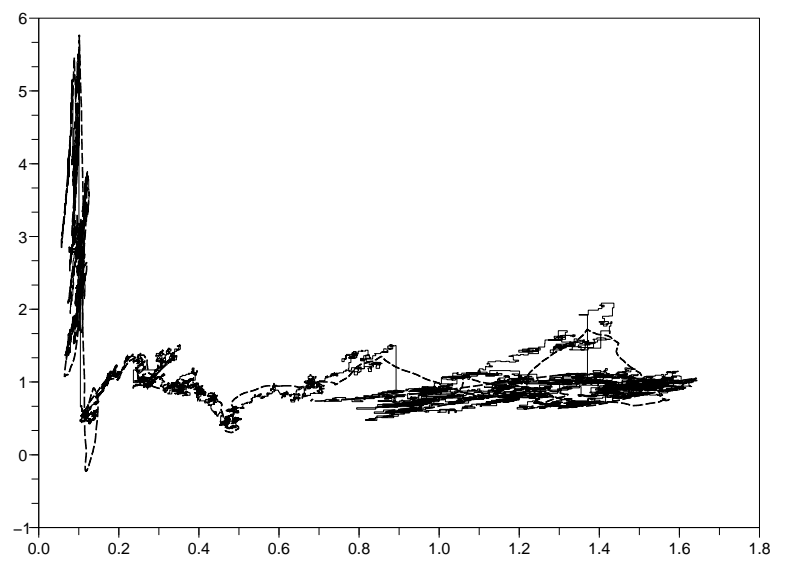

(a) $\mathfrak{C}$ : IBM (S\&P 500) (-) and its trend (- -)

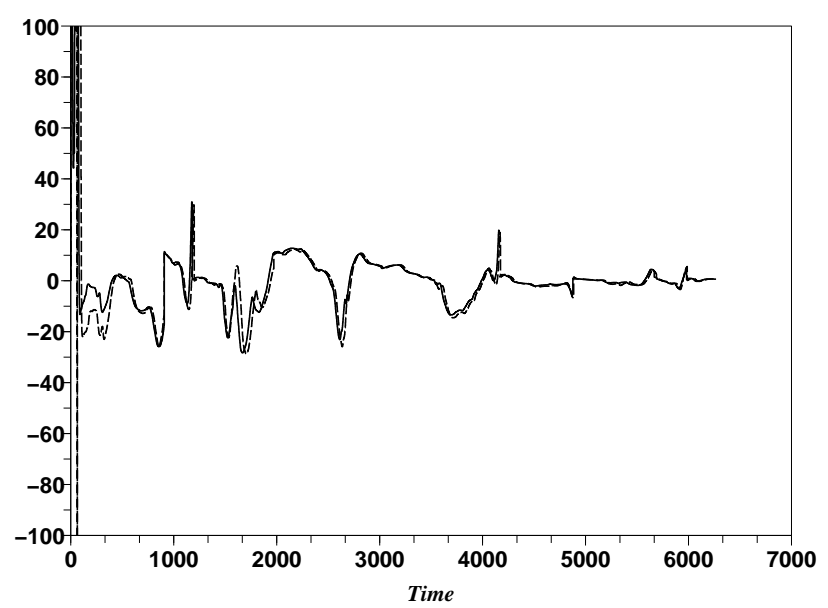

(b) JPM's $\beta(t)(-)$ and 20 days forcasting (- -)

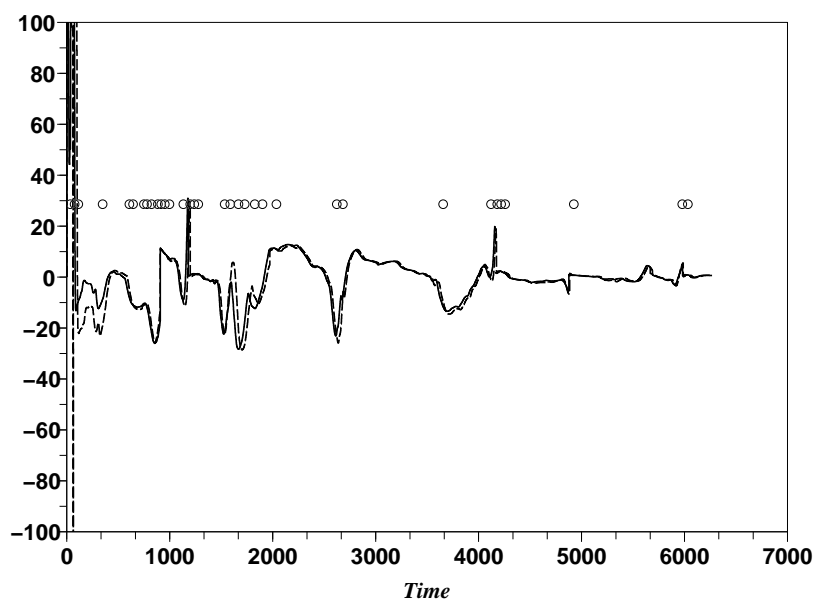

(d) 20 days forcasting of abrupt changes on JPM's $\beta(t)$ (o)

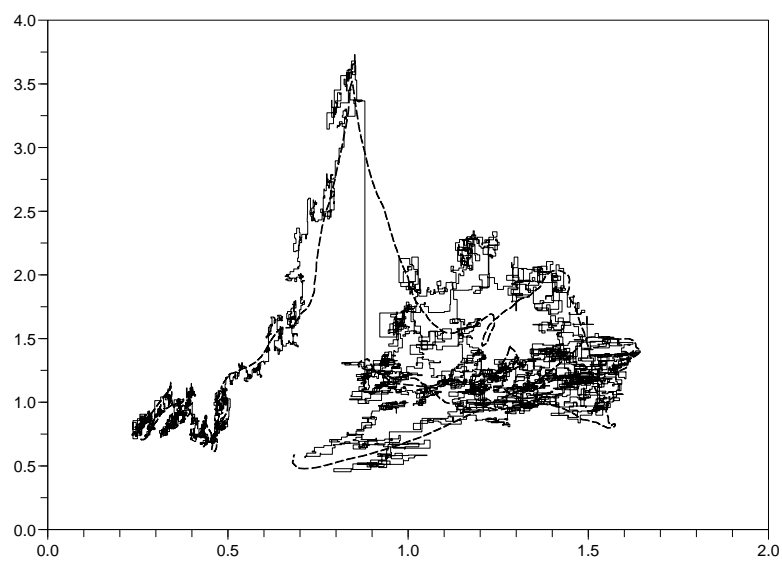

(b) $\mathfrak{C}$ : CCE (S\&P 500) (-) and its trend (- -)

Figure 4. Projection on the price plane 


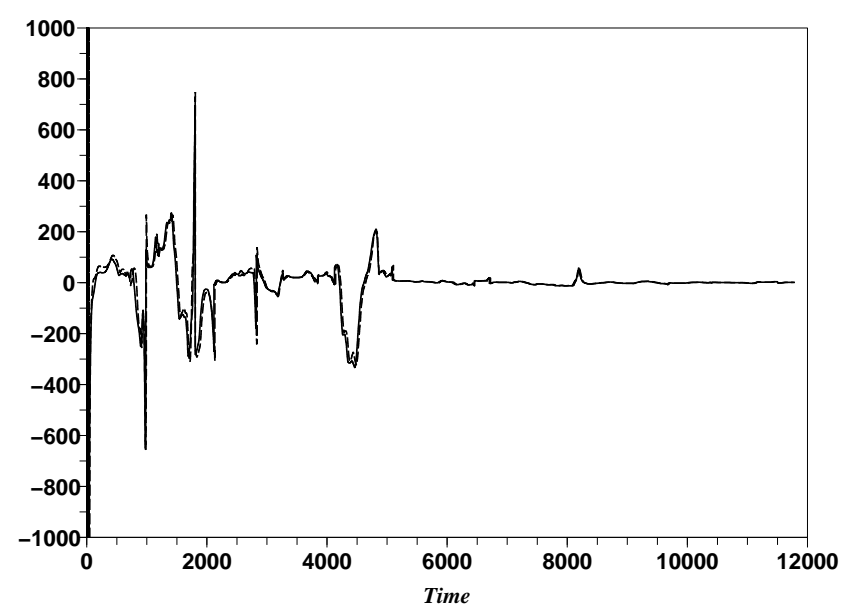

(a) IBM's $\beta(t)(-)$ and its forecast (- -)

Figure 5. 20 days forcasting

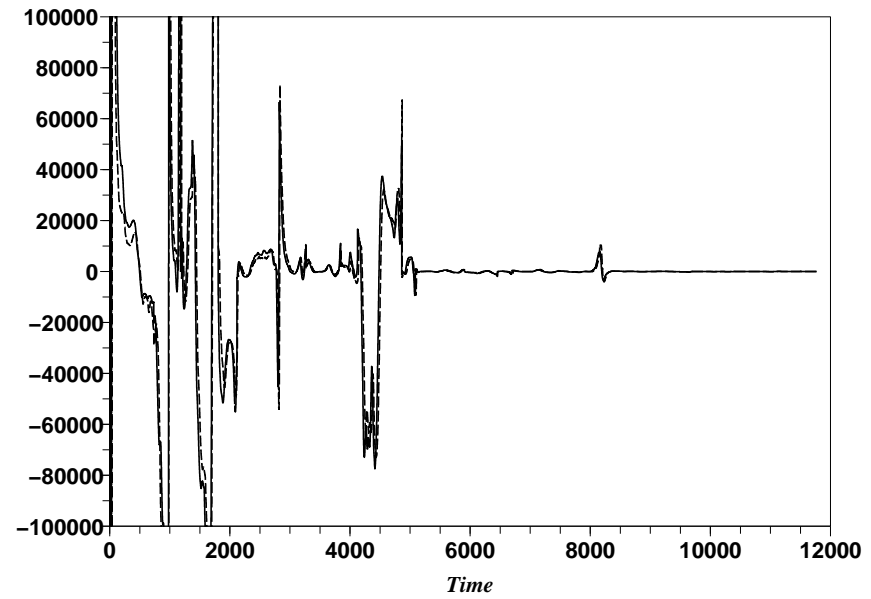

(a) IBM's $\frac{d \beta}{d x_{\mathfrak{C}}}(-)$ and its forecast (- -)

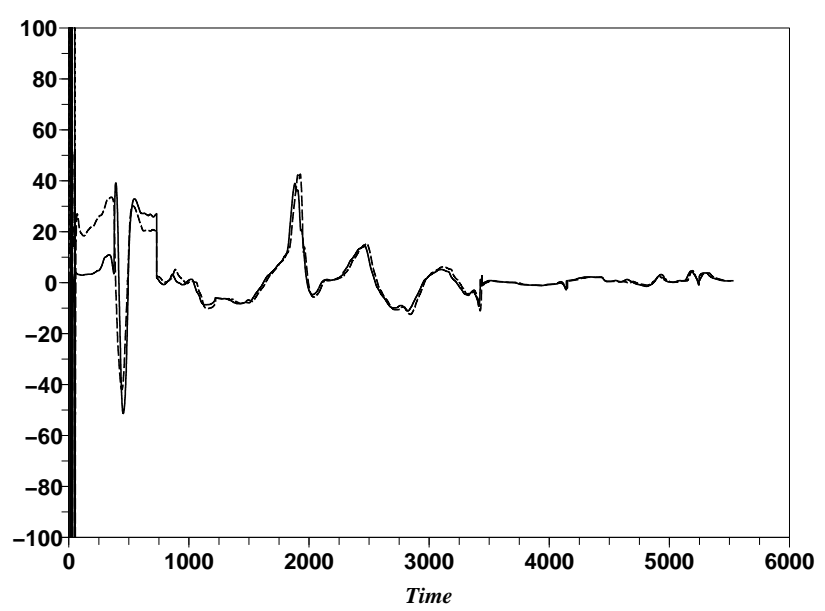

(b) CCE's $\beta(t)(-)$ and its forecast (- -)

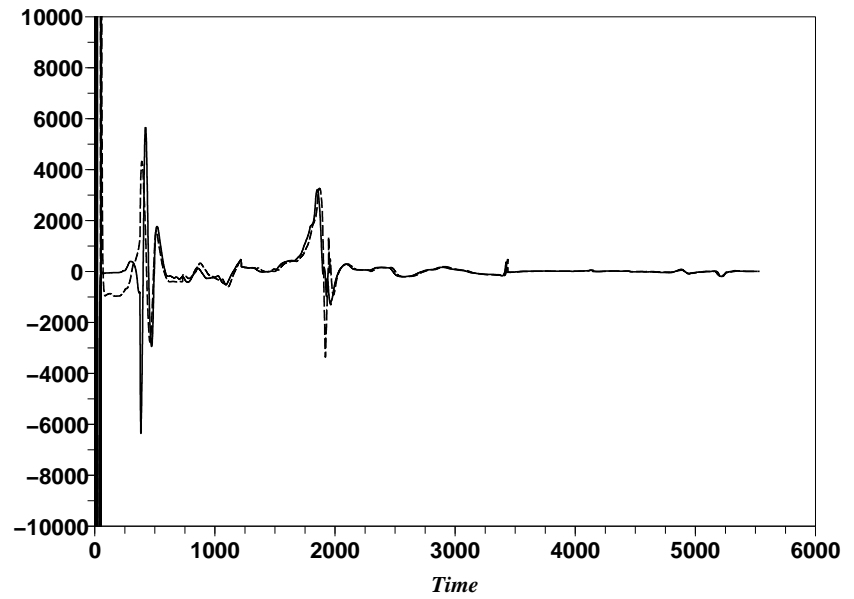

(b) CCE's $\frac{d \beta}{d x_{\mathfrak{C}}}(-)$ and its forecast (- -)

Figure 6. 20 days forcasting 